\title{
Patient Case Studies and Panel Discussion: Plasma Cell Neoplasms
}

\section{ABSTRACT}

Managing patients with plasma cell neoplasms, diseases in which abnormal plasma cells or myeloma cells form tumors in the bones or soft tissues of the body, poses numerous challenges for clinicians. At the NCCN 2019 Annual Congress: Hematologic Malignancies, a panel of experts discussed evidenced-based approaches for the treatment of patients with these diseases. Moderated by Dr. Andrew D. Zelenetz, the session focused on patients with transplant-ineligible newly diagnosed multiple myeloma, active multiple myeloma, and light chain amyloidosis.

J Natl Compr Canc Netw 2019;17(11.5):1437-1440 doi: $10.6004 /$ jnccn.2019.5034

Plasma cell neoplasms are diseases in which abnormal plasma cells or myeloma cells form tumors in the bones or soft tissues of the body. At the NCCN 2019 Annual Congress: Hematologic Malignancies, a panel of experts identified clinical challenges in managing patients with plasma cell neoplasms. Moderated by Dr. Andrew D. Zelenetz, the session focused on 3 case studies, which were used to develop an evidence-based approach for the treatment of these patients.

\section{Patient Case Study 1: Transplant-Ineligible, Newly Diagnosed Multiple Myeloma}

In the first case study, a 75-year-old man presented with new back pain and fatigue. The patient was able to perform all activities of daily living and resided approximately 70 miles away from the treatment center. Figure 1 shows results from imaging, laboratory tests, and bone marrow biopsy.

Although the patient did not harbor high-risk fluorescence in situ hybridization (FISH) changes, his lactate dehydrogenase (LDH) level was elevated at diagnosis. Elevated LDH as a risk factor has been associated with inferior outcomes, ${ }^{1,2}$ and is also factored into risk stratification by the Revised International Staging System (R-ISS) as the only nongenetic parameter, ${ }^{3}$ said Muhamed Baljevic, MD, University of Nebraska Medical Center.

Yvonne A. Efebera, MD, MPH, The Ohio State University Comprehensive Cancer Center - James Cancer Hospital and Solove Research Institute, noted that although this patient is transplant-ineligible, a 3-drug regimen is preferred over 2-drug options.

"This has been shown several times in newly diagnosed patients," said Dr. Efebera. "In the SWOG SO777 study, bortezomib with lenalidomide and dexamethasone showed better progression-free survival [PFS] and overall survival versus lenalidomide and dexamethasone alone. ${ }^{4}$ Furthermore, in the MAIA trial, the addition of daratumumab to lenalidomide and dexamethasone improved PFS versus lenalidomide and dexamethasone alone. ${ }^{5}$ However, because we don't have overall survival data from the MAIA trial, the triplet of bortezomib/ lenalidomide/dexamethasone is the only one to show survival benefit," she said.

Dr. Baljevic noted that bortezomib/lenalidomide/ dexamethasone is a category 1 NCCN recommendation for both transplant-eligible and transplant-ineligible patients based on the SWOG SO777 data. Although recent data from the ALYCONE trial suggest that quadruplet regimens may surpass triplet regimens, ${ }^{6}$ this particular induction combination may be more relevant to European practice patterns. The combination of daratumumab + bortezomib/melphalan/prednisone led to increased overall response rates and PFS. ${ }^{6}$ For transplant-eligible patients, transplant is still preferred to induce the deepest remission possible, Dr. Zelenetz noted, "but we're not seeing overall survival differences like we used to in the long-term."

This patient received bortezomib, lenalidomide, and dexamethasone and experienced a very good partial response after 6 cycles. The patient then switched to maintenance lenalidomide, $10 \mathrm{mg}$ daily. Complete responses encompass very deep remissions and clearly detectable disease, said Dr. Zelenetz, who noted that data have shown that undetectable minimal residual disease status is associated with better outcomes. ${ }^{7}$ Nevertheless, panelists agreed that minimal residual disease testing to guide therapy for this patient would not have be useful in clinical practice.

"This is a very expensive test, and to make it worthwhile, you want to be able do something with the information obtained," said Michael M. Green, MD, 
Radiograph: Multiple compression facture at $L 4$ and $L 5$

Laboratory results:

- Hemoglobin, $9.5 \mathrm{~g} / \mathrm{dL}$

- Creatinine, $1.2 \mathrm{mg} / \mathrm{dL}$

- Calcium, $10.6 \mathrm{mg} / \mathrm{dL}$

- Total protein, $11 \mathrm{~g} / \mathrm{dL}$

- SPEP $\geq$ M-protein, $4.2 \mathrm{~g} / \mathrm{dL}$

- IFE $\geq \lg G$ kappa

- KLC, 1,200 mg/L

- LLC, $5 \mathrm{mg} / \mathrm{L}$

- $\mathrm{k} / \mathrm{l}$ ratio:240

- Beta-2 microglobulin, $3.8 \mathrm{mg} / \mathrm{L}$

- LDH, 250 IU/L (199 ULN)

- Albumin, $3.7 \mathrm{~g} / \mathrm{dL}$

PET/CT scan:

- Multiple hypermetabolic lytic lesions

- No extramedullary disease noted

- Small level of FDG uptake 3.3-5.5 SUV throughout marrow of spine

Bone marrow biopsy:

- $80 \%$ cellularity

- 75\% kappa-restricted plasma cells

- FISH, t(11;14)

Figure 1. Results from laboratory, imaging, and biopsy studies for the patient in case study 1 .

Abbreviations: FISH, fluorescence in situ hybridization; IFE, immunofixation electrophoresis; KLC, kappa light chain; LDH, lactate dehydrogenase; SPEP, serum protein electrophoresis; SUV, standard uptake value; ULN, upper limit of normal.

Kaiser Permanente. “For this patient in particular, I don't think there was much utility."

\section{Early-Relapse Disease: No Clear Choice \\ for Treatment}

The patient received continuous maintenance therapy with lenalidomide. After 2 years of maintenance, he experienced toxicity from lenalidomide (diarrhea and fatigue) and took a treatment holiday. The first relapse occurred at a PFS time of approximately 39 months. Laboratory results showed a hemoglobin level of $10.2 \mathrm{~g} / \mathrm{dL}$ and a PET scan showed several new lytic bone lesions. Bone marrow biopsy showed $60 \%$ kappa plus plasma cells and FISH identified $t(14 ; 16), \operatorname{del}(17 p)$, and gain(1q) (4 copies).

Although many new agents are available for relapsed/ refractory multiple myeloma, panelists agree that triplet regimens remain the standard, with daratumumab combinations appearing quite effective. The sequencing of agents is still undefined, however. Several novel agents are in development, with early data on venetoclax and B-cell maturation antigen-targeted therapies showing promise.

"There are lots of positive trials and lots of options in this setting, but there is no clear, unequivocal correct answer, which is an important point," said Dr. Zelenetz.

"Oftentimes, when we're making a decision about what the next line of treatment will be at first relapse, we're really thinking about what the third-line option will be down the road," Dr. Green added. "Whatever decision we make up front is going to impact the kinds of options available later on."

\section{Patient Case Study 2: Active Multiple Myeloma}

In the second case study, a 53-year-old man presented with persistent swelling and pain around the left shoulder for several months. A small mass was detected on his left clavicle on physical examination. Medical history showed hypertension, schizoaffective disorder, and chronic hepatitis $\mathrm{B}$, and a diagnosis of active multiple myeloma was the panelists' consensus. Figure 2 shows test results for this patient. The patient was treated with lenalidomide, bortezomib, and dexamethasone (continued for 15 months). Approximately 1 month after starting treatment, he received radiotherapy to his left clavicle for 2 weeks.

Nina Shah, MD, UCSF Hellen Diller Family Comprehensive Cancer Center, noted that a carfilzomib + lenalidomide and dexamethasone (KRD) combination may have been the better option because of the patient's $17 \mathrm{p}$ deletion and high-risk disease.

"Data from the University of Chicago seem to show that when you gave patients at high risk more-aggressive induction of KRD followed by transplant and KRD consolidation and lenalidomide maintenance, their outcomes are similar to those with standard risk," said Dr. Shah. "That's a very small trial subset and there are a lot of caveats, but we have to do something different for patients at high risk than we're doing for those at standard risk."

Dr. Efebera added that for patients at high risk, 2-drug maintenance may yield better outcomes than single-agent maintenance. "We don't have any prospective studies, and only $15 \%$ of patients are high-risk, but retrospective analysis at Emory has shown that 2-drug maintenance is feasible and possibly better. The jury is still out," she said.

Dr. Baljevic noted that the field is moving toward 4-drug combinations for both standard- and high-risk disease, which raises legitimate questions regarding financial toxicity. "The possibility of moving toward pentads (5-drug combinations) with molecularly adapted regimens has even been discussed," he said. "Provided safety profiles would not be prohibitory, the idea would be to treat patients for a shorter period of time upfront, and see if less therapy in the long term is possible."

Although autologous stem cell transplantation (ASCT) was discussed, this patient elected not to move forward with it due to social concerns. Approximately 
1 month after stopping treatment, he experienced disease recurrence characterized by progressive disease in the sternum and rib.

"Patients may be initially resistant to transplant for a number of reasons, but after the disease comes back early, they may be more amenable," said Dr. Efebera, who noted that insurance companies are more frequently paying for collection of stem cells and storage.

This patient was started on daratumumab, pomalidomide, and dexamethasone, which he continued for 10 months before experiencing cytopenias with daratumumab and pomalidomide. Approximately 1 week after starting therapy, he received radiotherapy to the sternum (for 2 weeks).

"Radiotherapy should be used for symptomatic lesions and should not delay systemic therapy," said Dr. Zelenetz. He noted that 3 weeks after the patient started therapy, bone marrow biopsy showed $<5 \%$ atypical plasma cells. However, major complications and adverse events occurred during treatment, leading to drug disruptions and treatment delays. The patient's disease recurred 10 months after starting therapy. He was admitted for altered mental status, hypercalcemia, back pain, and was treated with carfilzomib, cyclophosphamide, and dexamethasone.

"There are other treatment options for extramedullary disease, but there really is no right answer," said Dr. Shah. "This patient should go to a clinical trial for CAR-T cell therapy or should undergo a transplant."

"For high-risk disease and for people who are young, undergoing transplant upfront is strongly recommended," added Dr. Green. "It could save the patient from getting into these situations."

- MRI (left shoulder) - Heterogeneously enhancing mass $(3.6 \times 2.3 \mathrm{~cm})$ clavicle

- Biopsy $\rightarrow$ plasmacytoma

- PET scan: Diffuse lytic lesions of the bone; large sternal mass $(5.2 \times 5.1 \mathrm{~cm}$; SUV 6.8)

- Labs: M-band: $3.4 \mathrm{~g} / \mathrm{dL}$; calcium: $9.4 \mathrm{mg} / \mathrm{dL}$; creatinine: $0.9 \mathrm{mg} / \mathrm{dL}$; hemoglobin: $12.2 \mathrm{~g} / \mathrm{dL}$; WBC: 3.4 ; platelets: 237; albumin: $3.6 \mathrm{gm} / \mathrm{dL}$; beta-2 microglobulin: $2.09 \mathrm{mg} / \mathrm{L}$

- Bone marrow aspiration and biopsy:

- $40 \%-50 \%$ involvement by atypical plasma cells

- Positive: CD138, CD38, dim/equivocal CD19, cytoplasmic kappa

- FISH: $17 p$ and $13 q$ deletion

Figure 2. Results from laboratory, imaging, and biopsy studies for the patient in case study 2.

Abbreviations: FISH, fluorescence in situ hybridization; SUV, standard uptake value.

\section{Patient Case Study 3: Light Chain Amyloidosis} For the final case study, a 63-year-old man presented with easy bruising, foamy urine, and red tongue, with no prior medical history. Figure 3 shows his test results.

Based on the diagnosis of light chain amyloidosis, panelists agreed that urine immunofixation should be performed for optimal diagnostic sensitivity. As Dr. Zelenetz reported, one study found that not performing urine immunofixation led to missing $6 \%$ of amyloidogenic clones. ${ }^{8}$

"For patients with light chain amyloidosis, I really want to stress the importance of mass spectroscopy in terms of identifying the clone and the type of amyloid," Dr. Efebera added. "It's been shown that $13 \%$ of patients with TTR [transthyretin] familial amyloidosis are treated as having light chain amyloidosis due to having monoclonal gammopathy of undetermined significance [MGUS] unrelated to their amyloidosis; $3 \%$ of the general population aged $\geq 50$ years have MGUS. It's very important that mass spectroscopy is incorporated into your tissue biopsy."

"Fat pad biopsies are easy and cheap," said Dr. Zelenetz. "If you're not sure, it's worth it to do a biopsy."

Following work-up, the Mayo Clinic Staging System for light chain amyloidosis was used. For those inexperienced with this system, Michaela Liedtke, MD, Stanford Cancer Institute, reassured the audience that this system is relatively straightforward and is routinely used in practice. "You basically plug in the numbers for the $N$-terminal pro-B-type natriuretic peptide and troponin, and the differential of the free light chains," she said. "The ratio is not as important as the difference between the involved light chain that produces the amyloid deposits and the uninvolved light chain."

"Although this is a prognostic tool, it also provides a lot of information about whether somebody is a transplant candidate or not," Dr. Baljevic explained. "After induction therapy and tumor reduction, recent data show that it's actually possible to make someone who was not initially a transplant candidate into a candidate." ${ }^{10}$

\footnotetext{
- Serum protein electrophoresis and immunofixation, lambda LC+

- Urine protein electrophoresis with immunofixation

- Free Lambda LC

- 24-hour protein excretion of $3 \mathrm{~g}$ (nonselective)

- FLC: Kappa 15 mg/L, lambda 170 mg/L

- Bone marrow:

- 45\% Lambda-restricted plasma cells

- Congo Red and thioflavin-T stains positive for amyloid

- Kidney biopsy and subsequent mass spectrometry: identified lambda-type AL amyloidosis
}

Figure 3. Results from laboratory and imaging studies for the patient in case study 3 . 
"Patients with amyloid differ significantly from patients with myeloma," Dr. Baljevic continued. "With light chain amyloidosis, it's even more important to try to get patients to transplant. It's one of the most important measures in terms of long-term outcomes that we can offer them." 11

This patient was treated with 4 cycles of cyclophosphamide, bortezomib, and dexamethasone and experienced a hematologic very good partial response. "Depth of response is important in myeloma, but even more important in amyloidosis because you want to reduce the light-chain burden as much as possible," said Dr. Liedtke.

According to Dr. Zelenetz, the key to making the diagnosis of light chain amyloidosis is suspecting the diagnosis in the first place. "This is the heart and soul of what we do as physicians," he concluded. "When you approach the patient, you have to have the right differential diagnosis. You've got to suspect, because you can't make the right diagnosis you're not thinking about."
Disclosures: Dr. Zelenetz has disclosed that he received consulting fees from AbbVie, Inc., Amgen Inc., AstraZeneca Pharmaceuticals LP, Celgene Corporation, Gilead Sciences, Inc., Janssen Pharmaceutica Products, LP, Novartis Pharmaceuticals Corporation, Adaptive Biotechnologies

Corporation, Genentech, Inc./Roche Laboratories, Inc., and Pharmacyclics; is a scientific advisor for AbbVie, Inc., AstraZeneca Pharmaceuticals LP, and MorphoSys AG; and receives grant/research support from BeiGene, Gilead Sciences, Inc., MEI Pharma Inc., and Roche Laboratories, Inc. Dr. Baljevic has disclosed that he received consulting fees from Cardinal Health and Takeda Pharmaceuticals North America, Inc., and served as an internal review committee member for Karyopharm Therapeutics and a scientific advisor for Takeda Pharmaceuticals North America, Inc. Dr. Efebera has disclosed that she has received honoraria from Janssen Pharmaceutica Products, LP, and Takeda Pharmaceuticals North America, Inc, and is a scientific advisor for Akcea Therapeutics. Dr. Green has disclosed that he has no financial interests, arrangement, affiliations, or commercial interests with any manufacturers of any products discussed in this article or their competitors. Dr. Liedtke has disclosed that she receives grant/research support from Agios, Inc., Amgen Inc., Celator Pharmaceuticals, Celgene Corporation, Genentech, Inc., Gilead Sciences, Inc., Janssen Pharmaceutica Products LP, bluebird bio, Inc., Prothena, Pfizer Inc., and Takeda Pharmaceuticals North America, Inc; received consulting fees from Amgen Inc.; and is a scientific advisor for Celgene Corporation, Janssen Pharmaceutica Products, LP, and Jazz Pharmaceuticals Inc. Dr. Shah has disclosed that she receives grant/research support from Celgene Corporation, Janssen Pharmaceutica Products, LP, bluebird bio, Inc., Sutro Biopharma; is a scientific advisor for Genentech, Inc., Seattle Genetics, Oncopeptides, Karoypharm, Surface Oncology, Precision Biosciences, Glaxo Smith Kline, Nektar, Amgen, Indapta Therapeutics, and Sanofi; and owns stock in Indapta Therapeutics.

\section{References}

1. Dimopoulos MA, Barlogie B, Smith TL, Alexanian R. High serum lactate dehydrogenase level as a marker for drug resistance and short survival in multiple myeloma. Ann Intern Med 1991;115: 931-935.

2. Terpos E, Katodritou E, Roussou M, et al. High serum lactate dehydrogenase adds prognostic value to the international myeloma staging system even in the era of novel agents. Eur J Haematol 2010; 85:114-119.

3. Palumbo A, Avet-Loiseau H, Oliva S, et al. Revised international staging system for multiple myeloma: a report from International Myeloma Working Group. J Clin Oncol 2015;33:2863-2869.

4. Durie BG, Hoering A, Abidi $\mathrm{MH}$, et al. Bortezomib with lenalidomide and dexamethasone versus lenalidomide and dexamethasone alone in patients with newly diagnosed myeloma without intent for immediate autologous stem-cell transplant (SWOG S0777): a randomised, open-label, phase 3 trial. Lancet 2017;389:519-527.

5. Facon $T$, Kumar S, Plesner $T$, et al. Daratumumab plus lenalidomide and dexamethasone for untreated myeloma. N Engl J Med 2019;380: 2104-2115.
6. Mateos MV, Dimopoulos MA, Cavo M, et al. Daratumumab plus bortezomib, melphalan, and prednisone for untreated myeloma. N Engl J Med 2018;378:518-528.

7. Munshi NC, Avet-Loiseau H, Rawstron AC, et al. Association of minimal residual disease with superior survival outcomes in patients with multiple myeloma: a meta-analysis. JAMA Oncol 2017;3: 28-35.

8. Dispenzieri A, Merlini G, Comenzo RL. Amyloidosis 2008 BMT tandem meetings (February 13-17, San Diego). Biol Blood Marrow Transplant 2008;14(Suppl 1):6-11.

9. Lachmann HJ, Booth DR, Booth SE, et al. Misdiagnosis of hereditary amyloidosis as AL (primary) amyloidosis. N Engl J Med 2002;346: 1786-1791.

10. Manwani R, Hegenbart U, Mahmood S, et al. Deferred autologous stem cell transplantation in systemic AL amyloidosis. Blood Cancer J 2018;8:101.

11. Dispenzieri A, Kyle RA, Lacy $M Q$, et al. Superior survival in primary systemic amyloidosis patients undergoing peripheral blood stem cell transplantation: a case-control study. Blood 2004;103: 3960-3963. 